\title{
LUMINOSITY INHOMOGENEITY OF QUASARS AND THE UPPER LIMIT OF THE DENSITY FLUCTUATION IN THE UNIVERSE
}

\author{
Yaoquan Chu \\ Center for Astrophysics \\ University of Science and \\ Technology of China \\ Hefei, China
}

\author{
Lizhi Fang \\ Beijing Astronomical Observatory \\ Beijing, China
}

\begin{abstract}
An upper limit to the amplitude of the overall density fluctuation has been found by means of the gravitational lensing effect of the density inhomogeneity on luminosity of quasars with large redshifts. The observed differences of luminosities of quasars located at different directions are partially given by the lensing effect; therefore, a useful upper limit to the inhomogeneity can be derived.
\end{abstract}

The propagation of radiation from distant sources will be affected by the metric perturbation of density inhomogeneity (Fang 1983). Therefore, the amplitude of the inhomogeneity can be found from the observed difference of luminosity between sources which originally have the same luminosity. Recently, the spatial distribution of quasars from many catalogues and surveys show that the distribution for high redshift quasars is uniform (Chu 1989). Since the lens effect is one of the reasons given for the observed difference or inhomogeneity of luminosity of quasars, an upper limit to the amplitude of inhomogeneity of dark matter can be derived, assuming that all the luminosity inhomogeneity of quasars comes from the lensing effect.

We developed a modified power-spectrum analysis method to find the required luminosity inhomogeneity. Its basic statistics are as follows:

$$
I_{U V}^{1}=\frac{2}{\sum m_{i}}\left|\sum_{i=1}^{n} m_{i} \exp \left[i\left(u \cdot x_{j}+v \cdot y_{j}\right)\right]\right|^{2},
$$

where $m_{i}$ is the magnitude of the $i$ th quasar, $u$ and $v$ are integers, and $x_{j}$ and $y_{j}$ are the coordinates of the $j$ th quasar. In order to increase the power of the test for luminosity inhomogeneity, we introduce the statistics $Q$

$$
Q=\frac{\sum I}{\sum v}
$$

with

$$
\sum I=\sum_{(u, v)} I_{U V}^{1} \text { and } \sum v=\frac{1}{n}\left[\sum_{i=1}^{n} m_{i}\right] \sum_{(u, v)} 2,
$$

where the sums in $\sum I$ and $\sum v$ are summed over all the $(u, v)$ with $\lambda_{U V}>\lambda_{c}$. If any inhomogeneity exists, $Q$ will deviate from unity and $(Q-1)$ can be used as a measure of the inhomogeneity amplitude. 
TABLE 1. The M-PSA Statistics for Quasars of Savage-Bolton Sample

\begin{tabular}{ccccccccccr}
\hline \hline $1 / \lambda_{c}$ & 1 & 2 & 3 & 4 & 5 & 6 & 7 & 8 & 9 & 10 \\
\hline $02^{\mathrm{h}},-50^{\circ}$ & 0.95 & 0.85 & 0.68 & 1.15 & 0.96 & 1.24 & 1.04 & 1.30 & 0.99 & 0.97 \\
$22^{\mathrm{h}},-18^{\circ}$ & 1.12 & 1.00 & 1.05 & 1.18 & 1.32 & 0.80 & 1.22 & 0.76 & 1.07 & 1.09 \\
\hline
\end{tabular}

The results of M-PSA for quasars with redshifts $z>2$ in the Savage-Bolton survey (Savage and Bolton 1979) are shown in Table 1. We note immediately from Table 1 that the mean of $(Q-1)$ is about 0.1 . The luminosity distribution of quasars with $z>2$ is uniform, especially for longer length scale, $1 / \lambda=1,2$, and 3 .

It appears that the mean luminosities of quasars located at different directions are the same to within about $10 \%$. From this we find that the upper limit to the total density inhomogeneity is

$$
\delta<\lambda_{10}^{-1 / 2} h_{0}^{-1 / 2} \Omega^{-1},
$$

where $\lambda_{10}$ is the length scale of the inhomogeneity in the unit of $10 \mathrm{Mpc}$. It seems to be less than the inhomogeneity amplitudes of galactic distribution by a factor of 3 to 5 . This conclusion should be considered as a very rough one. Nevertheless, this link between the distribution of quasars with $z>2$ and the large-scale inhomogeneity of dark matter merits our attention (Fang 1986).

\section{REFERENCES}

Fang, L.-Z. 1983, Scientia Sinica, 26, 297.

Chu, Y. and Zhu, X. 1989, Astro. Ap., in press.

Savage, A. and Bolton, J. 1979, MNRAS, 188, 599.

Fang, L.-Z., Chu, Y., and Zhu, X. 1986, Modern Physics Letters A, 1, 87.

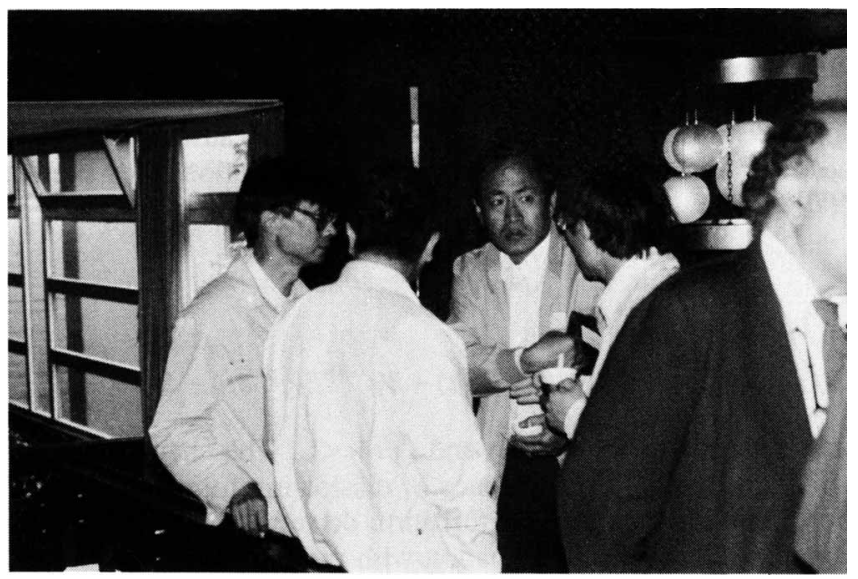

Zhong Yu Qian, Shengyin Wu, Xiang-Tao He, and Han Zou 